\title{
Direct effects of phenformin on metabolism/bioenergetics and viability of SH-SY5Y neuroblastoma cells
}

\author{
FINTAN GEOGHEGAN $^{1}$, NAOMI CHADDERTON ${ }^{2}$, G. JANE FARRAR ${ }^{2}$, \\ DANIELA M. ZISTERER ${ }^{1}$ and RICHARD K. PORTER ${ }^{1}$
}

\begin{abstract}
${ }^{1}$ School of Biochemistry and Immunology, Trinity Biomedical Sciences Institute; ${ }^{2}$ Ocular Genetics Unit, Smurfit Institute of Genetics, School of Genetics and Microbiology, Trinity College Dublin, Dublin 2, D02 R590, Republic of Ireland
\end{abstract}

Received May 10, 2016; Accepted February 13, 2017

DOI: $10.3892 / 01.2017 .6929$

\begin{abstract}
Phenformin, a member of the biguanides class of drugs, has been reported to be efficacious in cancer treatment. The focus of the current study was to establish whether there were direct effects of phenformin on the metabolism and bioenergetics of neuroblastoma SH-SY5Y cancer cells Cell viability was assessed using the alamar blue assay, flow cytometry analysis using propidium iodide and annexin $\mathrm{V}$ stain and poly (ADP-ribose) polymerase analysis. Cellular and mitochondrial oxygen consumption was determined using a Seahorse Bioscience Flux analyser and an Oroboros Oxygraph respirometer. Cells were transfected using electroporation and permeabilized for in situ mitochondrial functional analysis using digitonin. Standard protocols were used for immunoblotting and proteins were separated on denaturing gels. Phenformin was effective in reducing the viability of SH-SY5Y cells, causing $\mathrm{G}_{1}$ cell cycle arrest and inducing apoptosis. Bioenergetic analysis demonstrated that phenformin significantly decreased oxygen consumption in a dose- and time-dependent manner. The sensitivity of oxygen consumption in SH-SY5Y cells to phenformin was circumvented by the expression of NADH-quinone oxidoreductase 1 , a ubiquinone oxidoreductase, suggesting that complex I may be a target of phenformin. As a result of this inhibition, adenosine monophosphate protein kinase is activated and acetyl-coenzyme A carboxylase is inhibited. To the best of our knowledge, the current study is the first to demonstrate the efficacy and underlying mechanism by which phenformin directly effects the survival of neuroblastoma cancer cells.
\end{abstract}

Correspondence to: Professor Richard K. Porter, School of Biochemistry and Immunology, Trinity Biomedical Sciences Institute, Trinity College Dublin, 152-160 Pearse Street, Dublin 2, D02 R590, Republic of Ireland

E-mail: rkporter@tcd.ie

Key words: phenformin, neuroblastoma, SH-SY5Y cells, cancer, mitochondria, Ndi1

\section{Introduction}

Neuroblastoma is a malignancy that arises from the neural crest cells of the sympathetic nervous system and accounts for $15 \%$ of paediatric cancer mortalities (1). The cancer presents as a highly diverse heterogeneous disease that ranges from sporadically regressing to refractory forms (2). Although there have been advances in the understanding of this disease and the use of aggressive treatment strategies, including surgery, radiation therapy, immunotherapy and differentiation therapy, it remains one of the most challenging types of cancer to treat with a 5 -year survival rate of $42 \%$ (3). Therefore, there is a requirement for alternative approaches to treatment.

Drugs that target metabolic diseases, including the type II diabetes drugs metformin and phenformin, have been reported to be clinically important in the treatment of cancer $(4,5)$. Although phenformin was removed from the recommended treatment plan for diabetics as it has a propensity to induce lactic acidosis at high doses (6), it has been identified to have anti-neoplastic activity in phosphatase and tensin homolog (PTEN)-deficient tumors and in MCF-7 and MDA-MD-231 breast cancer cells $(7,8)$ at reduced concentrations compared with those established for efficacious treatment with metformin (9). Phenformin was also previously identified to induce the expression of the cyclin-dependent kinases inhibitor p21 and promote cell cycle arrest at the $\mathrm{G}_{2} / \mathrm{M}$ phase of the cell cycle leading to the apoptosis of neuroblastoma SH-SY5Y cells (10). However, the underlying mechanism by which phenformin causes these effects remains to be elucidated.

Adenosine monophosphate-activated protein kinase (AMPK) is a sensor of cellular homeostasis that is activated in response to a reducing level of cellular ATP in order to maintain the energy balance within the cell. AMPK is phosphorylated when the ratio of AMP:ATP decreases leading to the inhibition of ATP-consuming pathways and the activation of ATP-producing pathways $(11,12)$. AMPK phosphorylation promotes a phosphorylation cascade that regulates the activity of numerous downstream targets, including the mammalian target of rapamycin (mTOR) signalling pathway. In the majority of the cases where the biguanides, metformin and phenformin are associated with antitumor effects, it is considered that the AMPK/mTOR signalling pathway may serve an important role in the underlying mechanism of that action (7). 
Metformin and phenformin have been hypothesized to indirectly activate AMPK via the inhibition of complex I (13-15) of the respiratory chain in mitochondria therefore, decreasing ATP synthesis and increasing the AMP:ATP ratio $(13,14)$. The increase in peripheral glucose utilization and metabolism, and the stimulation of glycolytic lactate production, observed following metformin treatment of patients with diabetes, are effects that would be predicted with the inhibition of complex I of the respiratory chain (16).

Therefore, mitochondrial complex I has been a focus for investigating the activity of phenformin (and metformin). Wheaton et al (9) demonstrated that phenformin inhibits complex I of mitochondria at low concentrations and that this inhibition was important for its anti-proliferative effects on colon cancer cells. Furthermore, biguanide sensitivity of a range of cancer cells was reversed using ectopic expression of yeast NADH-quinone oxidoreductase 1 (Ndi1), a ubiquinone oxidoreductase that allows the bypassing of complex I function (17).

The present study demonstrated that phenformin may be capable of promoting cell death in human-derived neuroblastoma SH-SY5Y cells and may inform further on the underlying mechanism of this action.

\section{Materials and methods}

Reagents. Phenformin hydrochloride (Sigma Aldrich; Merck KGaA, Darmstadt, Germany) was dissolved in $\mathrm{H}_{2} \mathrm{O}$ and sterile filtered. Antimycin A (Sigma Aldrich; Merck $\mathrm{KGaA}$ ) was dissolved in ethanol. All the antibodies were sourced from Cell Signalling Technology, Inc., (Danvers, MA, USA). Media and FBS were obtained from Invitrogen (Thermo Fisher Scientific, Inc.). The Immobilon Western Chemiluminescence HRP substrate was sourced from Merck $\mathrm{KGaA}$. Bicinchoninic acid reagents were obtained from Pierce (Thermo Fisher Scientific, Inc.). The polyvinylidene difluoride (PVDF) membrane was purchased from Merck $\mathrm{KGaA}$, and the protease inhibitors were sourced from Roche Applied Science (Penzberg, Germany). Unless otherwise stated, chemicals were obtained from Sigma Aldrich (Merck $\mathrm{KGaA}$ ).

Cell culture. SH-SY5Y cells (American Type Culture Collection, Manassas, VA, USA) were maintained in Dulbecco's modified Eagle's medium (DMEM) F12 and GlutaMAX $^{\mathrm{TM}}$ medium supplemented with $2 \%$ penicillin/streptomycin and $10 \%$ fetal bovine serum. The cells were incubated at $37^{\circ} \mathrm{C}$ in a humidified atmosphere containing $5 \% \mathrm{CO}_{2}$ and passaged twice a week.

Alamar blue viability assay. The cell proliferation assay was performed using the AlamarBlue ${ }^{\circledR}$ assay (Medical Supply Company, Ltd., Dublin, Ireland) according to the manufacturer's protocol. The cells were plated at a density of $3 \times 10^{4}$ cells $/ \mathrm{ml}$ in triplicate in a $96-w e l l$ plate in a volume of $200 \mu \mathrm{l}$, cultured overnight at $37^{\circ} \mathrm{C}$ in $5 \% \mathrm{CO}_{2}$, and the following day the cells were treated with a range of concentrations of phenformin $(0.1-10,000 \mu \mathrm{M})$ and cultured for $72 \mathrm{~h}$. Alamar blue $(10 \% \mathrm{v} / \mathrm{v}, 20 \mu \mathrm{l})$ was added to the wells for 3-5 h. Fluorescence was analysed using Spectra Gemini microplate reader (Molecular Devices, LLC, Sunnyvale, CA, USA) at a wavelength of $544 \mathrm{~nm}$ with a reference wavelength of $590 \mathrm{~nm}$. Results were presented as the percentage of viability relative to the vehicle control (100\%). Dose response curves and $\mathrm{IC}_{50}$ determination were analyzed using Prism GraphPad version 5 software (GraphPad Software, Inc., La Jolla, CA, USA).

Cell cycle analysis by flow cytometry. On day 0, SH-SY5Y cells were plated in a 6 -well plate at a density of $1.1 \times 10^{5}$ cells/well. On day $1,80 \mu \mathrm{l}$ of phenformin, to a final concentration of 0.1 , $0.5,1,2$ and $5 \mathrm{mM}$, was added to the respective wells and incubated at $37^{\circ} \mathrm{C}$ in $5 \% \mathrm{CO}_{2}$ for $72 \mathrm{~h}$. Following treatment, the cells were trypsinised and pelleted by centrifugation. The cells were subsequently fixed in $70 \%$ ethanol/PBS and incubated overnight at $4{ }^{\circ} \mathrm{C}$. The ethanol was removed using centrifugation at $300 \mathrm{x} \mathrm{g}$ for $5 \mathrm{~min}$ at room temperature, and the pellets were resuspended in $400 \mu \mathrm{l}$ PBS supplemented with $10 \mu \mathrm{g} / \mathrm{ml}$ RNase A (Sigma Aldrich; Merck KGaA) and propidium iodide (PI) $100 \mu \mathrm{g} / \mathrm{ml}$ (Sigma Aldrich; Merck KGaA). The cells were then incubated in the dark for $30 \mathrm{~min}$ at $37^{\circ} \mathrm{C}$. Cell cycle distribution was analysed using the FACS Calibur flow cytometer (BD Biosciences, Franklin Lakes, NJ, USA) using Cell Quest Pro (version 4.3; BD Biosciences).

Annexin V assay. Following treatment, the cells were analysed using the Apopnexin Annexin V/Fluorescein isothiocyanate apoptosis kit (EMD Millipore, Billerica, MA, USA) following the manufacturer's protocol as previously described (18). The cells were analysed using the CyAn ADP Analyser flow cytometer (Beckman Coulter, Inc., Brea, CA, USA) and the Summit software (version 4.3; Beckman Coulter Inc.).

Oxygen consumption measurements. Oxygen consumption rates (OCR) were measured using the Oxygraph 2-k respirometer (Oroboros ${ }^{\circledR}$ Instruments Corporation, Innsbruck, Austria) and the XF24 Seahorse Extracellular Flux analyser (Agilent Technologies, Inc., Santa Clara, CA, USA).

Oxygraph measurements. SH-SY5Y cells were plated at a density of $1 \times 10^{6}$ in T75 flasks and were cultured overnight at $37^{\circ} \mathrm{C}$ in $5 \% \mathrm{CO}_{2}$. The following day, the cells were treated with $1 \mathrm{mM}$ phenformin or vehicle $\left(\mathrm{H}_{2} \mathrm{O}\right)$ for $6-48 \mathrm{~h}$, trypsinized and pelleted by centrifugation at $300 \mathrm{x}$ g for $5 \mathrm{~min}$ then resuspended in the appropriate cell culture media and divided equally between the two chambers in the oxygraph respirometer. Once stable basal cellular oxygen consumption rates were observed, the mitochondrial electron transport chain inhibitor antimycin $\mathrm{A}(1 \mu \mathrm{M})$ was added to the two chambers using a Hamilton syringe to determine cellular oxygen consumption rates not due to oxidative phosphorylation.

Seahorse measurements. SH-SY5Y cells were plated at a density of $8 \times 10^{5}$ in the Agilent Seahorse XF24 cell culture micro-plate (Agilent Technologies, Inc.) and incubated overnight at $37^{\circ} \mathrm{C}$ in $5 \% \mathrm{CO}_{2}$. The cells were subsequently incubated at $37^{\circ} \mathrm{C}$ in $5 \%$ $\mathrm{CO}_{2}$ with $1 \mathrm{mM}$ phenformin or vehicle for 0.5-6 h and treated with a range of concentrations of phenformin $(0.05-2 \mathrm{mM})$ for $6 \mathrm{~h}$ for the dose response experiment. Subsequently, $30 \mathrm{~min}$ prior to the assay, the media was replaced with $500 \mu 1$ of XF assay medium (Seahorse Bioscience; Agilent Technologies 
GmbH) supplemented with $17 \mathrm{mM}$ glucose (Sigma Aldrich; Merck KGaA). Mitochondrial oxygen consumption was obtained by subtracting the Antimycin A OCR from the basal OCR.

NADH dehydrogenase transfection. As the origin of the Ndi1 gene is from yeast, the Ndil gene was modified to include human codons and therefore, optimize the expression of the Ndil gene in human cells, including human-derived neuroblastoma SH-SY5Y cells. The Ndil construct used in this study had an altered codon usage profile so that it was compatible with human codon usage, which differs from yeast. The optimised sequence was synthesized de novo (Invitrogen; Thermo Fischer Scientific, Inc.) and cloned into the pAAV-MCS plasmid (Agilent Technologies GmbH, Waldbronn, Germany) using Xba1 (catalog no. R0145) and Xho1 (catalog no. R0146) (both New England BioLabs Inc., Ipswich, MA, USA) restriction enzymes for $2 \mathrm{~h}$ at $37^{\circ} \mathrm{C}$. Fragments were isolated on a $1 \%$ agarose gel and ligated together using T4 DNA ligase (Roche Applied Science) at $18^{\circ} \mathrm{C}$ overnight. Following transformation into XLI-blue competent cells (Agilent Technologies GmbH), DNA was isolated (Qiagen GmbH, Hilden, Germany), and the sequences of the DNA fragments were verified by sequencing (Eurofins Genomics, GmbH, Ebersberg, Germany). Ndi1 and control pAAV-MCS vectors (containing green fluorescent protein) were transfected into SH-SY5Y cells using the Neon electroporation system (Invitrogen; Thermo Fisher Scientific, Inc.). Transfection conditions were optimised for maximum expression of the plasmid. The cells were subsequently plated at a density of $4 \times 10^{5}$ cells in the Seahorse micro-plates, incubated at $37^{\circ} \mathrm{C}$ in $5 \% \mathrm{CO}_{2}$ for $48 \mathrm{~h}$ and treated with a range of concentrations of phenformin (0.1-1 mM) for $6 \mathrm{~h}$.

Digitonin permeabilisation of SH-SY5Y cells. SH-SY5Y cells were plated at a density of $8 \times 10^{5}$ in the Seahorse microplates and incubated overnight at $37^{\circ} \mathrm{C}$ in $5 \% \mathrm{CO}_{2}$. Half of the SH-SY5Y cells were incubated with $100 \mu \mathrm{M}$ phenformin for $6 \mathrm{~h}$ and $30 \mathrm{~min}$ prior to the assay, the DMEM F12 media was replaced by mitochondrial assay buffer $[220 \mathrm{mM}$ mannitol, $70 \mathrm{mM}$ sucrose, $10 \mathrm{mM} \mathrm{K \textrm {K } _ { 2 } \mathrm { PO } _ { 4 } , 5 \mathrm { mM } \mathrm { MgCl }}$, $2 \mathrm{mM}$ 4-(2-hydroxyethyl)-1-piperazineethanesulphonic acid, $1.0 \mathrm{mM}$ EGTA and $0.2 \%(\mathrm{w} / \mathrm{v})$ fatty acid-free bovine serum albumin, pH 7.4, supplemented with 4 mM ADP (Sigma Aldrich; Merck KGaA) and permeabilized with 10 or $50 \mu \mathrm{g} / \mathrm{ml}$ digitonin (Sigma Aldrich; Merck KGaA)]. Concentrations of digitonin were previously optimised in the laboratory. Basal rates were measured according to the previously described protocol by Divakaruni et al (19), then complex I substrates (glutamate/malate; $5 \mathrm{mM}$; Sigma Aldrich; Merck KGaA) and complex II substrate (succinate (Sigma Aldrich; Merck KGaA), $5 \mathrm{mM}$ ) were added and the increase in OCR was recorded.

Western blot analysis. SH-SY5Y cells were harvested in AMPK Lysis buffer [50 mM Tris/ $\mathrm{HCl} \mathrm{pH} 7.4$ at $4^{\circ} \mathrm{C}, 50 \mathrm{mM}$ NaF, $5 \mathrm{mM}$ Na pyrophosphate, $1 \mathrm{mM}$ EDTA, $1 \mathrm{mM}$ EGTA, $250 \mathrm{mM}$ mannitol, $1 \%$ (v/v) Triton X-100, $1 \mathrm{mM}$ dithiothreitol (DTT) and a proteinase inhibitors cocktail tablet], snap frozen and stored at $-20^{\circ} \mathrm{C}$ until required as previously performed by Hardie et al (20). For poly(ADP-ribose) polymerase (PARP) analysis, the cells were suspended in PARP lysis buffer [62.5 mM Tris (pH 6.8), 6M urea, 2\% (w/v) SDS, $10 \%$ (v/v) glycerol, $0.00125 \%$ (w/v) bromophenol blue and $50 \mathrm{mM}$ DTT] with the volume amended to the size of the pellet. The cells were sonicated for $10 \mathrm{~min} 40 \mathrm{kHz}$ in a water bath (Sonicator 2000; Branson Ultrasonics Co., Danbury $\mathrm{CT}$, USA) to ensure complete lysis and boiled at $100^{\circ} \mathrm{C}$ for $5 \mathrm{~min} .5 \%(\mathrm{w} / \mathrm{v})$ DTT was added to the samples prior to being loaded in a 10\% SDS-PAGE gel immersed in electrolyte in an electrophoresis apparatus (BIORAD Mini-Protean; Bio-Rad Laboratories, Inc., Hercules, CA, USA). Equal quantities of protein $(50 \mu \mathrm{g})$ (as determined by bicinchoninic acid assay, Pierce; Thermo Fisher Scientific, Inc.) were separated on 6 or $10 \%$ SDS-PAGE gels and transferred to PVDF membranes. The membranes were blocked in 5\% (w/v) Marvel ${ }^{\circledR}$ milk in Tris-buffered saline (TBS)-Tween for $1 \mathrm{~h}$ at room temperature. The membranes were incubated with primary antibody at $4^{\circ} \mathrm{C}$ overnight, washed at room temperature and incubated in horseradish peroxidise conjugated secondary antibody for $1 \mathrm{~h}$ at room temperature and washed again at room temperature. Western blot analysis was performed using antibodies targeting AMPK $\alpha$ (1:1,000; catalog no. 2532L), phospho-AMPK (1:714; catalog no. 2531L), acetyl-coenzyme A carboxylase (ACC) (1:714; catalog no. 3662S), phospho-ACC $(1: 1,000$; catalog no. 3661S; Cell Signalling Technology, Inc.), $\gamma$-tubulin (1:2,000; catalog no. NB110-90616; Novus Biologicals, Ltd., Cambridge, UK), Ndi1 (custom made; 1:500; Cambridge Research Biochemicals, Cleveland, UK), $\beta$-actin (1:5,000; catalog no. AB8227), (Abcam, Cambridge, UK) and monoclonal antibodies to PARP $(1: 1,000$; catalog no. AM30; Merck $\mathrm{KGaA}$ ) followed by incubation with horseradish peroxidase (HRP)-conjugated anti-mouse (1:2,500; catalog no. W4021), anti-rabbit (1:2,500; catalog no. W4011; Promega Corporation, Madison, IW, USA). Immobilon Western Chemiluminescence HRP substrate (EMD Millipore) was used for protein detection. Western blots were normalised to total AMPK or ACC as a control and densitometry analysis of bands was performed using ImageJ software (version 1.47v; National Institutes of Health, Bethesda, MD, USA). The increase in phosphorylation was presented as the fold change compared with the control.

Statistical analysis. Unless otherwise stated, data are expressed as the mean \pm standard error of the mean from three separate experiments performed in triplicate. Statistical analysis was performed using an unpaired Student's t-test. $\mathrm{P}<0.05$ was considered to indicate a statistically significant difference.

\section{Results}

Phenformin reduces the viability of SH-SY5Y cells in a dose-dependent manner. To evaluate the potency of phenformin on the neuroblastoma SH-SY5Y cell line, cells were treated with vehicle $\left(\mathrm{H}_{2} \mathrm{O}\right)$ or with a range of concentrations of phenformin (0.1-10 mM) for $72 \mathrm{~h}$. As presented in Fig. 1A, phenformin decreased the cell viability in a dose-dependent manner with maximum cytotoxic effect at $5 \mathrm{mM}$. The $\mathrm{IC}_{50}$ value for SH-SY5Y cells treated with phenformin was $2.76 \pm 0.09 \mathrm{mM}$.

Phenformin induces $G_{l}$ cell cycle arrest followed by apoptosis in a dose-dependent manner. A transient increase in the $\mathrm{G}_{1}$ population relative to the vehicle control was observed at 

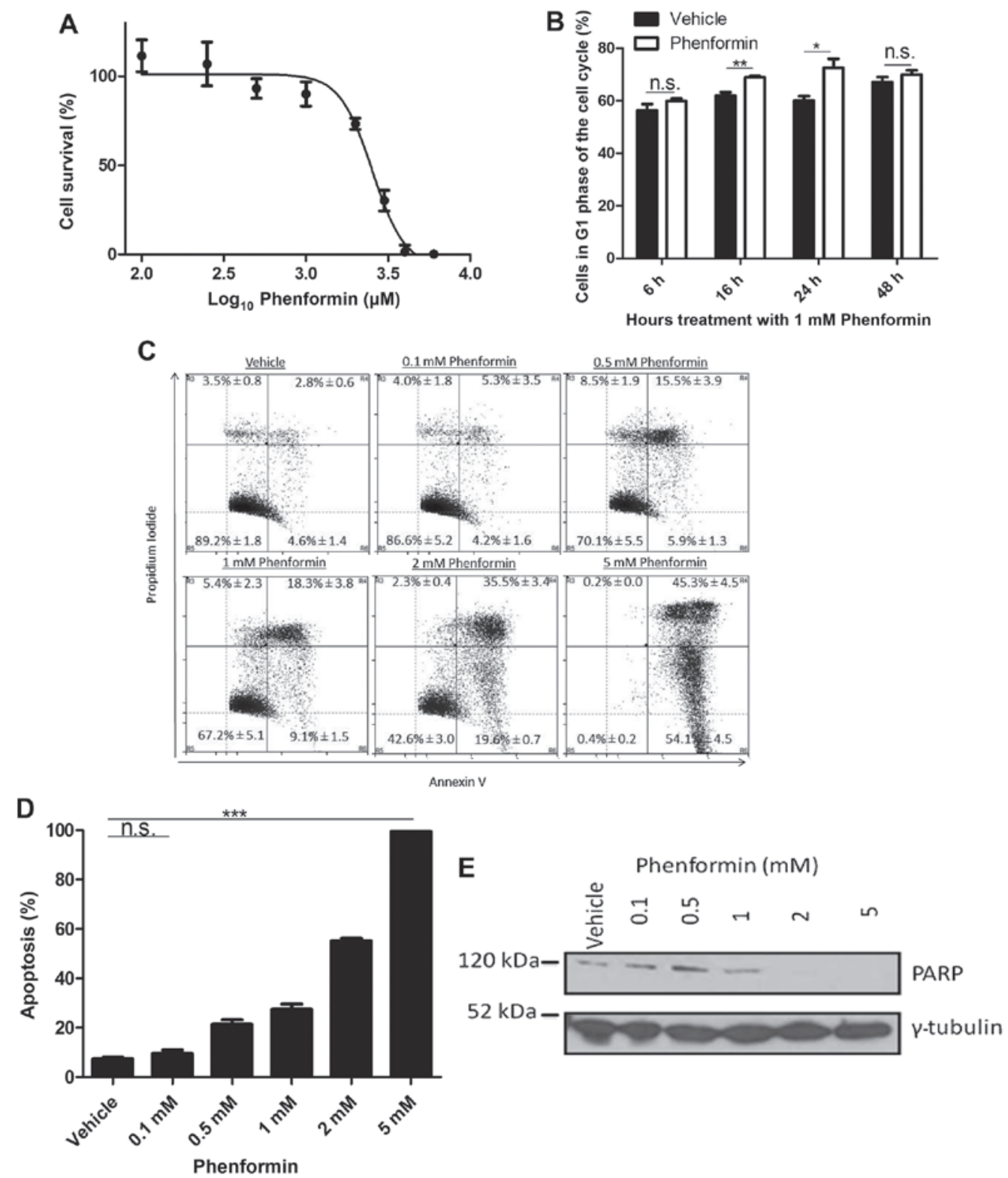

Figure 1. Phenformin reduces the viability, induces $\mathrm{G}_{1}$ cell cycle arrest and induces apoptosis in SH-SY5Y neuroblastoma cells. (A) SH-SY5Y cells were

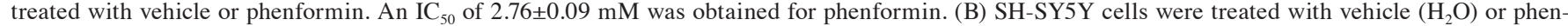
formin for the times points indicated. Cells were stained with PI and analysed by flow cytometry for the percentage of cells in $\mathrm{G}_{1}$ phase of the cell cycle. (C, D) SH-SY5Y cells were treated with phenformin and stained with PI and Annexin V. (E) Immunoblot demonstrating the reduction in PARP in SH-SY5Y cells with increasing phenformin concentration. Data are presented as the standard deviation between the mean percentage of cells undergoing the cell death. PARP, poly(ADP-ribose) polymerase. ${ }^{*} \mathrm{P}<0.05,{ }^{* *} \mathrm{P}<0.001,{ }^{* * * *} \mathrm{P}<0.0001$, n.s., not significant.

$16 \mathrm{~h}(\mathrm{P}<0.005)$ and $24 \mathrm{~h}(\mathrm{P}<0.05$; Fig. 1B). This significant increase indicated that the cells were undergoing $\mathrm{G}_{1}$ cell cycle arrest as a result of phenformin treatment. To validate that cell death occurred as a result of phenformin treatment at the $72 \mathrm{~h}$ time point and to determine the mechanism of cell death, the cells were stained with PI and annexin V. It was observed that as the percentage of live cells decreased, there was an increase in the population of cells undergoing apoptosis as a result of phenformin treatment (Fig. 1C), which demonstrated a significant difference at the dose of $0.5 \mathrm{mM}$ phenformin compared with the vehicle $(\mathrm{P}<0.0001$; Fig. 1D). A reduction was observed in PARP protein with increasing phenformin concentrations (Fig. 1E). These results demonstrate that phenformin may be promoting $\mathrm{G}_{1}$ cell cycle arrest prior to the induction of apoptosis.
Phenformin inhibits cellular/mitochondrial oxygen consumption in SH-SY5Y cells. The ability of phenformin to affect the metabolism of the cells in suspension was examined. As shown in Fig. 2A, there was significant inhibition of total cellular OCR at $6 \mathrm{~h}(\mathrm{P}<0.0001), 16 \mathrm{~h}(\mathrm{P}<0.005), 24 \mathrm{~h}(\mathrm{P}<0.0001)$ and $48 \mathrm{~h}(\mathrm{P}<0.0001)$ following phenformin $(1 \mathrm{mM})$ treatment of SH-SY5Y cells. Correcting for non-mitochondrial OCR, it was found that phenformin demonstrated a complete inhibition of mitochondrial oxygen consumption at all time points (Fig. 2B).

Following the determination that phenformin was affecting the mitochondrial OCR as early as $6 \mathrm{~h}$, the present study investigated whether phenformin affected oxygen consumption at earlier time points. Adherent cells were treated with $1 \mathrm{mM}$ phenformin and the OCR was measured using a Seahorse Flux Analyser with correction using non-mitochondrial OCR. 

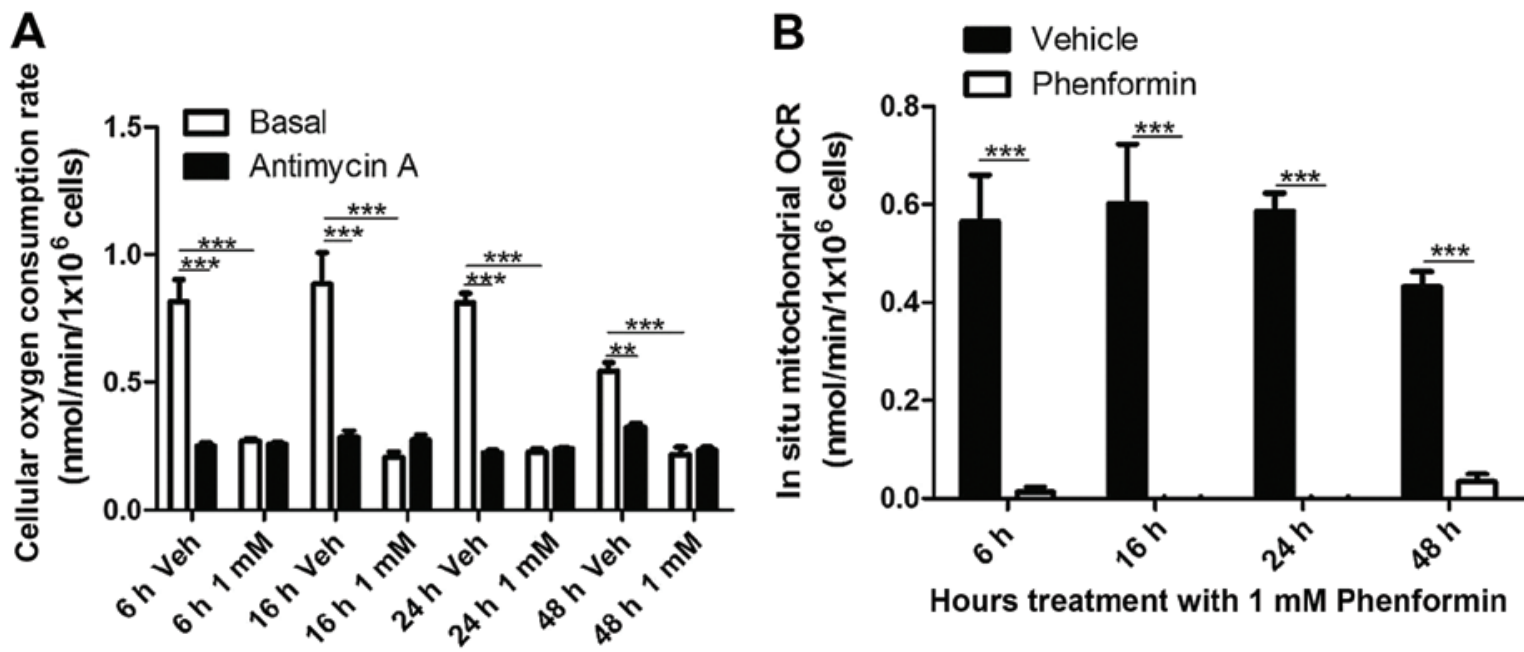

Figure 2. Time-dependent inhibition of oxygen consumption in SH-SY5Y cells in suspension as determined using an oxygraph respirometer. Cells were treated with vehicle or phenformin and placed in an oxygraph respirometer containing F12 Dulbecco's modified Eagle's media. (A) Total cellular OCR. (B) In situ mitochondrial OCR (cellular-antimycin A) within SH-SY5Y cells. OCR, oxygen consumption rate. Data is represented as the mean \pm standard error. "P<0.05, ${ }^{* *} \mathrm{P}<0.001,{ }^{* * *} \mathrm{P}<0.0001$.

A

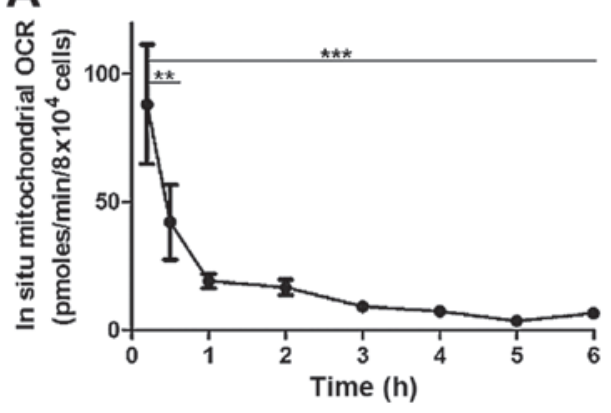

B

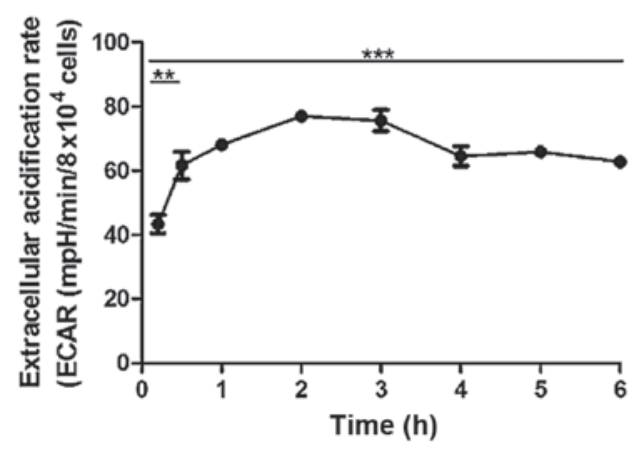

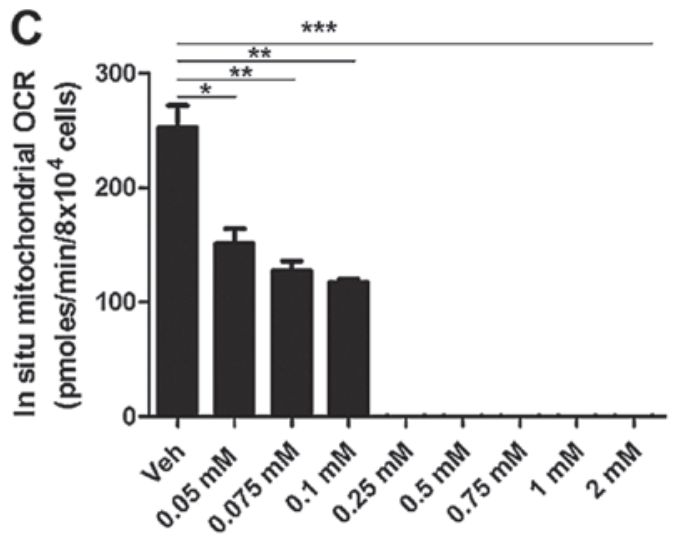

Phenformin

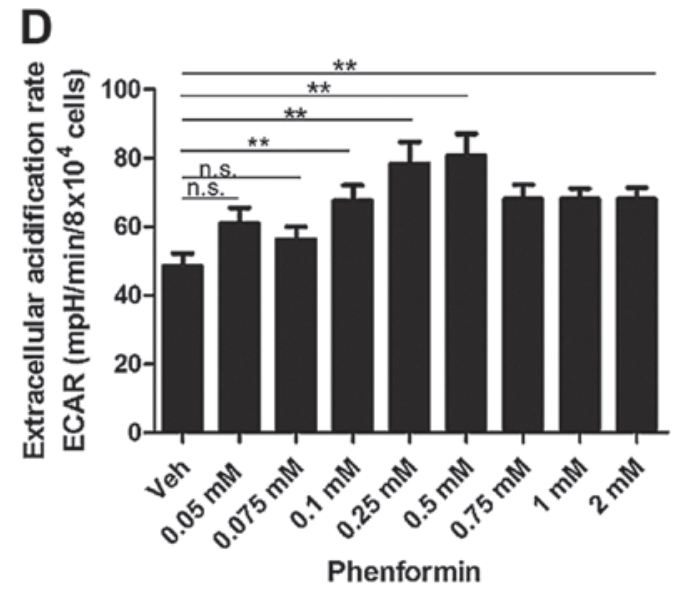

Figure 3. Phenformin inhibits OCR and increases ECAR in adherent SH-SY5Y cells as determined using the Seahorse Flux analyser. A Seahorse Flux analyser was used to measure time dependent inhibition of (A) OCR and (B) ECAR and concentration dependent inhibition of (C) mitochondrial OCR and (D) cellular ECAR of SH-SY5Y cells. OCR, oxygen consumption rate; ECAR, extracellular acidification rate; Data is represented as the mean \pm standard error. " $\mathrm{P}<0.05$, ${ }^{* *} \mathrm{P}<0.001,{ }^{* * *} \mathrm{P}<0.0001$. n.s., not significant.

There was a significant decrease in mitochondrial OCR from $30 \mathrm{~min}(\mathrm{P}<0.005)$ with a time-dependent decrease continuing until $6 \mathrm{~h}$ of incubation $(\mathrm{P}<0.0001)$ following treatment with $1 \mathrm{mM}$ phenformin (Fig. 3A). Concordant with this decrease in cellular OCR, there was a significant increase in the extracellular acidification rate (ECAR) from as early as $30 \mathrm{~min}$. This increase in ECAR remained statistically significant up to the $6 \mathrm{~h}$ timepoint $(\mathrm{P}<0.0001)$ (Fig. 3B). 

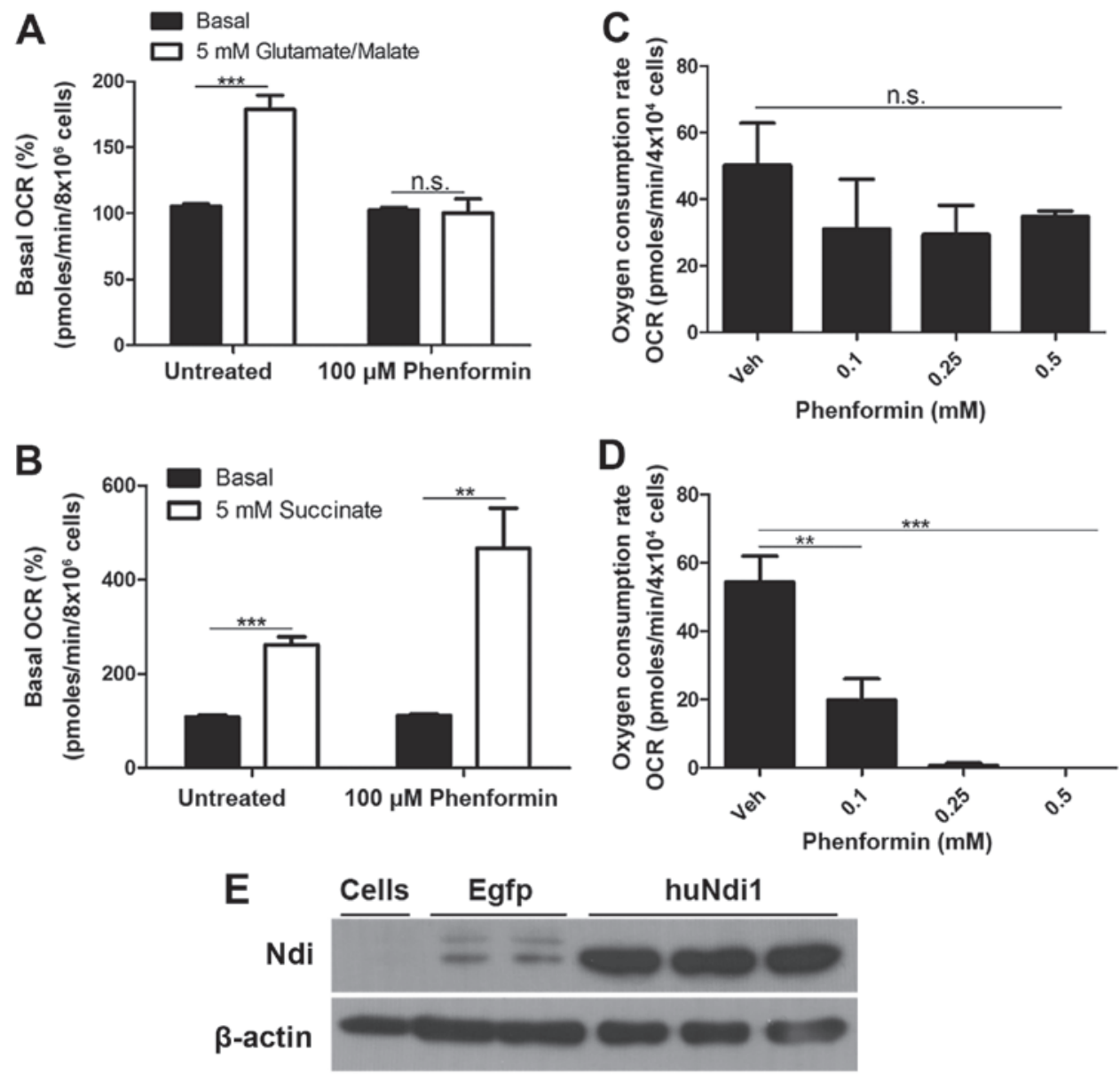

Figure 4. Identification of complex I as the target of phenformin inhibition in mitochondrial oxygen consumption. OCR in SH-SY5Y cells was measured using (A) glutamate/malate (generating substrates for complex I) and (B) succinate (substrate for complex II) in the presence and absence of phenformin. Independently, OCR was measured in SH-SY5Y cells treated with phenformin transfected with (C) an empty vector GFP tagged pAAV-MCS plasmid or (D) a pAAV-MCS plasmid encoding humanised Ndi1 and (E) confirmation that huNdi1 was expressed in SH-SY5Y cells using the pAAV-MCS plasmid encoding huNdi1, but not in cells containing GFP tagged pAAV-MCS plasmid (Egfp) or non-transfected cells (Cells). OCR, oxygen consumption rate; n.s., not significant; GFP, green fluorescent protein. Data is represented as the mean \pm standard error. ${ }^{*} \mathrm{P}<0.05,{ }^{* *} \mathrm{P}<0.001,{ }^{* * *} \mathrm{P}<0.0001$.

At a concentration of $0.05 \mathrm{mM}$ phenformin $(\mathrm{P}<0.05)$ there was a significant reduction in the in situ mitochondrial OCR and at a concentration of $0.25 \mathrm{mM}$ phenformin $(\mathrm{P}<0.0005)$ exhibited complete inhibition of mitochondrial OCR (Fig. 3C). There was a significant increase in the ECAR at $0.1 \mathrm{mM}$ phenformin $(\mathrm{P}<0.005)$, which persisted up to $1 \mathrm{mM}$ phenformin $(\mathrm{P}<0.005)$ (Fig. 3D). The current study also demonstrated that phenformin decreases intracellular reactive oxygen-containing species production in a concentration-dependent manner (data not shown).

Inhibition of mitochondrial complex I of SH-SY5Y cells by phenformin. To determine whether the inhibition of mitochondrial OCR by phenformin was a result of the inhibition of complex I, mitochondrial OCR was measured in digitonin permeabilized SH-SY5Y cells. In the presence of ADP and complex I substrates (glutamate/malate), phenformin demonstrated a complete inhibition of any increase in oxygen consumption as a result of the addition of glutamate and malate (Fig. 4A). However, phenformin did not inhibit the increase in mitochondrial OCR in digitonin-permeabilised SH-SY5Y cells following the addition of succinate, a complex II substrate (Fig. 4B). These experiments identified that phenformin inhibits mitochondrial OCR via complex I, but not complex II. Further evidence that the inhibition of in situ mitochondrial oxygen consumption by phenformin occurs at complex I was demonstrated by recovery experiments in which the inhibition at complex I is circumvented by transfection with Ndi1. SH-SY5Y cells treated with a number of concentrations of phenformin (0.1-0.5 mM) and were transiently transfected with an empty vector or a version of the yeast Ndi1 gene optimized for expression in human cells. Ndi1 is a single subunit of the NADH dehydrogenase, that oxidizes $\mathrm{NADH}$ in a similar process present in mammalian complex I, without proton pumping or ROS generation (21). The expression of Ndil conferred resistance to the inhibitory effects of phenformin (Fig. 4C) compared with cells transfected with an empty vector (Fig. 4D). The confirmation of Ndil protein expression was demonstrated using an immunoblot (Fig. 4E).

AMPK and ACC activation in SH-SY5Y cells occurs as a result of treatment with phenformin over a time course determined using western blot analysis. Following the demonstration that phenformin inhibits complex I of the mitochondria, it was hypothesized that there would be an increase in AMP and ADP levels, therefore activating AMPK, the major energy 
A

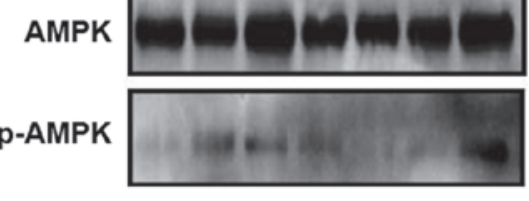

B

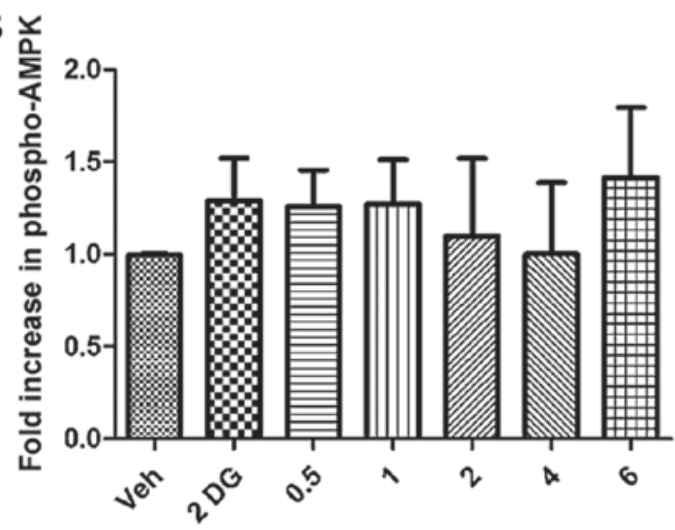

Treatment with $1 \mathrm{mM}$ Phenformin (h)

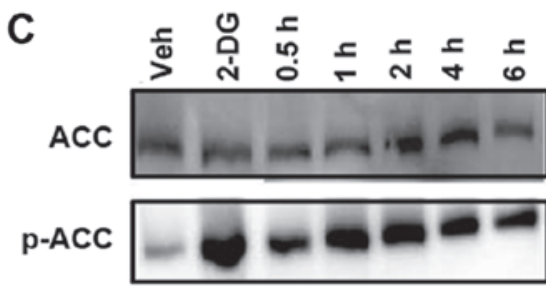

D o

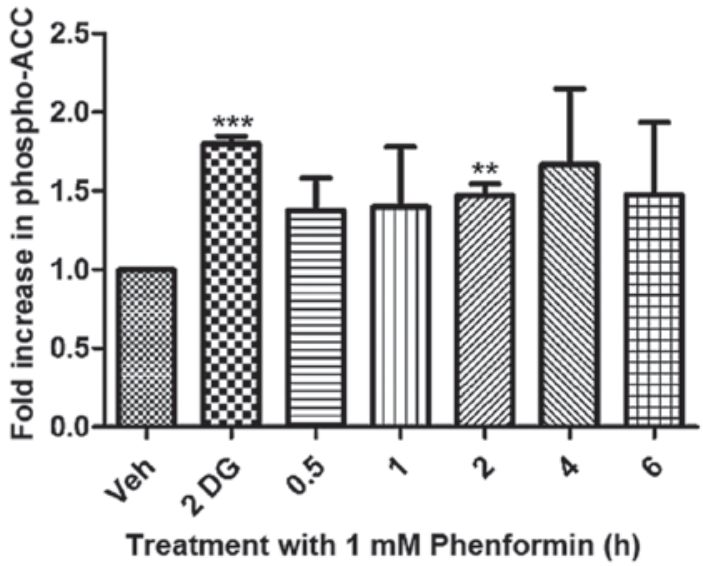

Figure 5. Phenformin treatment-dependent phosphorylation of AMPK and ACC as determined by immunoblot analysis. Whole cell lysates (50 $\mu \mathrm{g}$ protein) were prepared from confluent cultures of SH-SY5Y cells and separated by SDS-PAGE gels and transferred to a polyvinylidene fluoride membranes. (A) Representative blot of AMPK activation. (B) Fold increase in p-AMPK relative to vehicle, normalised against loading control for AMPK. (C) Representative blot of the phosphorylation of ACC. (D) Fold increase in p-ACC relative to vehicle, normalised against loading control ACC. Data is represented as the mean \pm standard error. ${ }^{*} \mathrm{P}<0.05,{ }^{* *} \mathrm{P}<0.001,{ }^{* * *} \mathrm{P}<0.0001$. AMPK, adenosine monophosphate-activated protein kinase; -AMPK, phosphorylation of adenosine monophosphate-activated protein kinase; ACC, acetyl-CoA carboxylase; p-ACC, phosphorylation of acetyl-CoA carboxylase; 2-DG, 2-deoxyglucose; Veh, vehicle.

sensor of the cell. In addition, the current study investigated ACC, which is a downstream enzyme phosphorylated by AMPK as a further indication of AMPK activation.

Activation (via phosphorylation) of AMPK in SH-SY5Y cells occurred as a result of phenformin treatment compared with the vehicle-treated controls (Fig. 5A and B). The inhibition (via AMPK phosphorylation) of ACC also occurs as a result of phenformin treatment compared with the vehicle-treated controls and this is significantly different after $2 \mathrm{~h}$ of phenformin treatment $(\mathrm{P}<0.05)$ (Fig. $5 \mathrm{C}$ and $\mathrm{D})$. The positive control 2-deoxyglucose increased the phosphorylation of AMPK and ACC as expected.

\section{Discussion}

Neuroblastoma is a paediatric malignancy that affects the sympathetic nervous system. Patients are initially responsive to treatment, including etoposide, carboplatin, vincristine and doxorubicin, but become refractory $(22,23)$ due to development of multi-drug resistance (24). Resistance may develop as a result of numerous underlying mechanisms, such as loss of p53, amplification of the MYCN oncogene and increased expression of drug efflux pumps (25). There is a requirement for alternative treatments for these resistant forms of neuroblastoma.

Phenformin, a former type II diabetic drug, has demonstrated significant anti-neoplastic activity in humans and animals $(7,10,17)$ at lower concentrations compared with its analogue metformin. Lactic acidosis, a phenformin-associated adverse effect, is insufficient to be considered as contraindicative as a cancer therapy due to the marked anti-neoplastic activity demonstrated by phenformin treatment $(26,27)$. Phenformin is less polar compared with metformin and therefore, has increased lipid solubility (28). Phenformin is able to permeate across biological membranes and has been identified to have a higher affinity for mitochondrial membranes compared with metformin (29). The molecular mechanisms underlying the antitumor effects of phenformin (and metformin) remain to be elucidated. It has been hypothesized that the anti-cancer effect of metformin may be as a result of pleiotrophic effects via insulin and insulin growth factors (28). The focus of this study was to determine whether phenformin directly promotes death of SH-SY5Y cancer cells and if so, to determine the underlying mechanism of this action.

The current study determined the effect of phenformin on the viability of the neuroblastoma SH-SY5Y cells. The $\mathrm{IC}_{50}$ for phenformin was identified to be in a similar range to that of SKOV3 and HEY ovarian cells (30), Sk-Mel-28 and WM115 melanoma cells (31) and MCF-7 and MDA-MB-231 breast cells (32). In the present study, metformin did not reduce the viability of the neuroblastoma SH-SY5Y cells to a level $<50 \%$, and thus, an $\mathrm{IC}_{50}$ value was not obtained (results not shown). Similar results for metformin were observed by Caraci et al (10) in the same cell line. Caraci et al (10) also demonstrated a lower $\mathrm{IC}_{50}$ value $(500 \mu \mathrm{M})$ following phenformin treatment of SH-SY5Y cells compared with the results of the present study $(2.76 \pm 0.09 \mathrm{mM})$. This differential may be as result of methodological variation, such as the use of scraping to detach cells and the use of the MTT assay to determine viability in Caraci et al (10), compared with the use of trypsin to harvest cells and the Alamar blue assay to evaluate 
cell viability in the present study. Reassuringly, however, this previous study is concordant with the present finding that phenformin is capable of directly affecting neuroblastoma cells by inducing cell death.

Phenformin has been identified to transiently increase the expression of the cell cycle inhibitor p21 and to cause $\mathrm{G}_{1}$ and $\mathrm{G}_{2} / \mathrm{M}$ arrest in a cell type-specific manner $(10,30)$. The current study has demonstrated that phenformin causes an accumulation of cells in the $\mathrm{G}_{1}$ phase of the cell cycle prior to inducing cell death. By contrast, Caraci et al (10) demonstrated that phenformin caused $\mathrm{G}_{2} / \mathrm{M}$ arrest in SH-SY5Y cells and this discrepancy may be as a result of methodological variation, such as their use of scraping to detach cells compared with the use of trypsin to detach cells or differences in cell storage prior to FACS analysis $\left(4^{\circ} \mathrm{C}\right.$ vs. $\left.-20^{\circ} \mathrm{C}\right)$ and sample preparation prior to FACS analysis (pre-incubated samples with RNase A for $1 \mathrm{~h}$ prior to adding PI compared with treating with RNase A and PI, simultaneously).

Investigation into the underlying mechanism of cell death induced by phenformin treatment of the SH-SY5Y cells exhibited a substantial increase in the apoptotic population of cells. These results are in accordance with previous studies that have demonstrated phenformin to promote apoptosis in A549 non-small cell lung cancer cells, MC7 and MDA-MD-231 breast cancer cells and PTEN-deficient tumors $(7,8,33)$.

The current study also demonstrated that phenformin significantly inhibits in situ mitochondrial oxygen consumption at subcytotoxic micromolar concentrations as well as increasing glycolytic flux in a dose- and time-dependent manner in neuroblastoma cells. These data are concordant with the observations of Owen et al (14) who demonstrated the potent inhibition of mitochondrial oxygen consumption by phenformin in a micromolar range in hepatoma cells. This previous study suggested that the positive charge of phenformin allows it to accumulate within the matrix of mitochondria as a result of the membrane potential $(34,35)$. El-Mir et al (13) also demonstrated a time-dependent effect of phenformin on oxygen consumption in hepatocytes. Taken together, these data suggest that the underlying mechanism of action of phenformin may be at the level of mitochondrial function.

Additionally, the current study found that phenformin inhibited complex I substrates, but not complex II substrates, through the promotion of mitochondrial OCR in permeabilized SH-SY5Y neuroblastoma cells. Furthermore, the expression of 'humanized' yeast Ndil protected the non-permeabilised SH-SY5Y cells from the inhibitory effects of phenformin on mitochondrial OCR. Therefore, phenformin appears to inhibit complex I. The results of the present study are in accordance with previous studies that have suggested that phenformin has antitumor effects via the inhibition of complex I in cancer cell lines $(9,17)$.

As phenformin inhibits complex I, the present study aimed to determine whether AMPK was activated. A previous study suggested that phenformin does not act directly on AMPK but indirectly through increasing intracellular AMP and ADP concentrations (36). These findings are in agreement with the observation that phenformin does not activate AMPK in cells that have a mutation in AMPK that effects AMP and ADP sensitivity (20). Whether phenformin is required to activate AMPK to affect the cells proliferative or survival capacity has yet to be elucidated. Huang et al (7) identified that AMPK activation was important in decreasing the occurrence of PTEN-deficient tumors following phenformin treatment, whereas Shackelford et al (33) found that phenformin was effective in the treatment of LKB1-deficient non-small cell lung cancer, as LKB1 is the major upstream kinase activating AMPK. In the present study, the activation of AMPK and inhibition of ACC, a downstream target of AMPK, occurs as a result of phenformin treatment.

In conclusion, phenformin is effective in reducing the cell viability of the human-derived neuroblastoma SH-SY5Y cancer cells through the promotion of $G_{1}$ cell cycle arrest and apoptosis. Bioenergetic analysis found that phenformin significantly decreased mitochondrial oxygen consumption at sub-cytotoxic concentrations in a dose- and time-dependent manner via the inhibition of the mitochondrial complex I while simultaneously increasing glycolytic flux. As a result of this inhibition by phenformin, AMPK is activated and ACC inhibited. Therefore, phenformin may have potential as an effective anti-cancer agent.

\section{Acknowledgements}

RKP and FG would like to thank the Higher Education Authority PRTLI5 scheme (grant no. 12259) for funding the project. GJF and NC wish to acknowledge Science Foundation Ireland (SFI) (grant no. 11/PI/1080) for the funding of their work.

\section{References}

1. Galenkamp K, Carriba P, Urresti J, Planells-Ferrer L, Coccia E, Lopez-Soriano J, Barneda-Zahonero B, Moubarak RS, Segura MF and Comella JX: TNFa sensitizes neuroblastoma cells to FasL-, cisplatin- and etoposide-induced cell death by NF- $\kappa B$-mediated expression of Fas. Mol Cancer 14: 62, 2015.

2. Amoroso F, Capece M, Rotondo A, Cangelosi D, Ferracin M, Franceschini A, Raffaghello L, Pistoia V, Varesio L and Adinolfi E: The $\mathrm{P} 2 \mathrm{X} 7$ receptor is a key modulator of the PI3K/GSK3 $\beta /$ VEGF signaling network: Evidence in experimental neuroblastoma. Oncogene 34: 5240-5251, 2015.

3. Cheung NK and Dyer MA: Neuroblastoma: Developmental biology, cancer genomics and immunotherapy. Nat Rev Cancer 13: 397-411, 2013

4. Dowling RJ, Goodwin PJ and Stambolic V: Understanding the benefit of metformin use in cancer treatment. BMC Med 9: 33, 2011.

5. Evans JM, Donnelly LA, Emslie-Smith AM, Alessi DR and Morris AD: Metformin and reduced risk of cancer in diabetic patients. BMJ 330: 1304-1305, 2005.

6. Kwong SC and Brubacher J: Phenformin and lactic acidosis: A case report and review. J Emerg Med 16: 881-886, 1998.

7. Huang X, Wullschleger S, Shapiro N, McGuire VA, Sakamoto K, Woods YL, McBurnie W, Fleming S and Alessi DR: Important role of the LKB1-AMPK pathway in suppressing tumorigenesis in PTEN-deficient mice. Biochem J 412: 211-221, 2008.

8. Appleyard MV, Murray KE, Coates PJ, Wullschleger S, Bray SE, Kernohan NM, Fleming S, Alessi DR and Thompson AM: Phenformin as prophylaxis and therapy in breast cancer xenografts. Br J Cancer 106: 1117-1122, 2012.

9. Wheaton WW, Weinberg SE, Hamanaka RB, Soberanes S, Sullivan LB, Anso E, Glasauer A, Dufour E, Mutlu GM, Budigner GS and Chandel NS: Metformin inhibits mitochondrial complex I of cancer cells to reduce tumorigenesis. Elife 3: e02242, 2014.

10. Caraci F, Chisari M, Frasca G, Chiechio S, Salomone S, Pinto A, Sortino MA amd Bianchi A: Effects of phenformin on the proliferation of human tumor cell lines. Life Sci 74: 643-650, 2003.

11. Martin M and Marais R: Metformin: A diabetes drug for cancer, or a cancer drug for diabetics? J Clin Oncol 30: 2698-2700, 2012. 
12. Carling D: The AMP-activated protein kinase cascade-a unifying system for energy control. Trends Biochem Sci 29: 18-24, 2004.

13. El-Mir MY, Nogueira V, Fontaine E, Avéret N, Rigoulet M and Leverve X: Dimethylbiguanide inhibits cell respiration via an indirect effect targeted on the respiratory chain complex I. J Biol Chem 275: 223-228, 2000.

14. Owen MR, Doran E and Halestrap AP: Evidence that metformin exerts its anti-diabetic effects through inhibition of complex 1 of the mitochondrial respiratory chain. Biochem J 348 Pt 3: 607-614, 2000

15. Miskimins WK, Ahn HJ, Kim JY, Ryu S, Jung YS and Choi JY: Synergistic anti-cancer effect of phenformin and oxamate. PLoS ONE 9: e85576, 2014.

16. Bailey CJ and Turner RC: Metformin. N Engl J Med 334: 574-579, 1996.

17. Birsoy K, Possemato R, Lorbeer FK, Bayraktar EC, Thiru P, Yucel B, Wang T, Chen WW, Clish CB and Sabatini DM: Metabolic determinants of cancer cell sensitivity to glucose limitation and biguanides. Nature 508: 108-112, 2014.

18. Zhang $X$, Wang $H$, Zhang S, Song J, Zhang Y, Wei X and Feng Z: MiR-134 functions as a regulator of cell proliferation, apoptosis and migration involving lung septation. In Vitro Cell Dev Biol Anim 48: 131-136, 2012.

19. Divakaruni AS, Rogers GW and Murphy AN: Measuring mitochondrial function in permeabilized cells using the seahorse XF analyzer or a clark-type oxygen electrode. Curr Protoc Toxicol 60 25.2: 1-16, 2014

20. Hardie DG, Salt IP and Davies SP: Analysis of the role of the AMP-activated protein kinase in the response to cellular stress. Methods Mol Biol 99: 63-74, 2000.

21. Seo BB, Kitajima-Ihara T, Chan EK, Scheffler IE, Matsuno-Yagi A and Yagi T: Molecular remedy of complex I defects: Rotenone-insensitive internal NADH-quinone oxidoreductase of Saccharomyces cerevisiae mitochondria restores the NADH oxidase activity of complex I-deficient mammalian cells. Proc Natl Acad Sci USA 95: 9167-9171, 1998.

22. Seeger RC and Reynolds CP: Treatment of high-risk solid tumors of childhood with intensive therapy and autologous bone marrow transplantation. Pediatr Clin North Am 38: 393-424, 1991.

23. Keshelava N, Seeger RC, Groshen S and Reynolds CP: Drug resistance patterns of human neuroblastoma cell lines derived from patients at different phases of therapy. Cancer Res 58: 5396-5405, 1998.

24. Lennon JC, Bright SA, Carroll E, Butini S, Campiani G, O'Meara A Williams DC and Zisterer DM: The novel pyrrolo-1,5-benzoxazepine, PBOX-6, synergistically enhances the apoptotic effects of carboplatin in drug sensitive and multidrug resistant neuroblastoma cells. Biochem Pharmacol 87: 611-624, 2014.
25. Balayssac D, Cayre A, Authier N, Bourdu S, Penault-Llorca F, Gillet JP, Maublant J, Eschalier A and Coudore F: Patterns of P-glycoprotein activity in the nervous system during vincristine-induced neuropathy in rats. J Peripher Nerv Syst 10: 301-310, 2005.

26. Shackelford DB and Shaw RJ: The LKB1-AMPK pathway: Metabolism and growth control in tumour suppression. Nat Rev Cancer 9: 563-575, 2009.

27. Goodarzi MO and Bryer-Ash M: Metformin revisited: Re-evaluation of its properties and role in the pharmacopoeia of modern antidiabetic agents. Diabetes Obes Metab 7: 654-665, 2005.

28. Rizos CV and Elisaf MS: Metformin and cancer. Eur J Pharmacol 705: 96-108, 2013

29. Hawley SA, Pan DA, Mustard KJ, Ross L, Bain J, Edelman AM, Frenguelli BG and Hardie DG: Calmodulin-dependent protein kinase kinase-beta is an alternative upstream kinase for AMP-activated protein kinase. Cell Metab 2: 9-19, 2005.

30. Jackson AL, Kilgore JE, Qiu H, Zhou C, Gehrig PA and Victoria L: Abstract 2442: Antitumorigenic effects of phenformin in human ovarian cancer cell lines. Cancer Res 73: 2442, 2013.

31. Yuan P, Ito K, Perez-Lorenzo R, Del Guzzo C, Lee JH, Shen CH, Bosenberg MW, McMahon M, Cantley LC and Zheng B: Phenformin enhances the therapeutic benefit of BRAF(V600E) inhibition in melanoma. Proc Natl Acad Sci USA 110: 18226-18231, 2013

32. Guo Z, Chavez KJ, Alvarez J, Zhang X, Norris B, Maher M, Morgan M, Schumacher RJ, Cuellar R, Sevrioukova IF, et al: Abstract 2689: Breast cancer inhibition by a novel and potent biguanide, N1-hexyl-N5-benzyl-biguanide. Cancer Res 74: 2689, 2014.

33. Shackelford DB, Abt E, Gerken L, Vasquez DS, Seki A, Leblanc M, Wei L, Fishbein MC, Czernin J, Mischel PS and Shaw RJ: LKB1 inactivation dictates therapeutic response of non-small cell lung cancer to the metabolism drug phenformin. Cancer Cell 23: 143-158, 2013.

34. Davidoff F: Effects of guanidine derivatives on mitochondrial function: III. The mechanism of phenethylbiguanide accumulation and its relationship to in vitro respiratory inhibition. J Biol Chem 246: 4017-4027, 1971.

35. Bridges HR, Jones AJ, Pollak MN and Hirst J: Effects of metformin and other biguanides on oxidative phosphorylation in mitochondria. Biochem J 462: 475-487, 2014.

36. Hardie DG: AMPK: A target for drugs and natural products with effects on both diabetes and cancer. Diabetes 62: 164-2172, 2013. 